\title{
INTENSIDAD Y DURACIÓN DEL ESTRÉS TÉRMICO EN VERANO EN EL ÁREA URBANA DE MADRID
}

\author{
Alberto Martí Ezpeleta \\ alberto.marti@usc.es \\ Dominic Royé \\ dominic.roye@usc.es \\ Departamento de Geografía, \\ Universidad de Santiago, \\ Plaza da Universidade, 1 \\ 15782 Santiago de Compostela
}

\begin{abstract}
Resumen: En este trabajo se aplica una metodología nueva al estudio de las noches calurosas, también denominadas "tropicales", en el área metropolitana de Madrid, de cara a evaluar desde una perspectiva temporal y espacial aquellas noches en las que la población pueda verse afectada por estrés térmico. La utilización de dos indicadores obtenidos a través de datos horarios, junto a la información climática suministrada por el modelo UrbClim, ha permitido conocer a una escala de detalle las características térmicas de las noches del mes de julio entre 2008 y 2017, pudiendo así evaluar con más precisión el riesgo para el bienestar y la salud de la población. Los resultados muestran una gran variabilidad interurbana en cuanto a intensidad y duración del estrés térmico, así como una correlación significativa entre las intensidades de la isla de calor y los índices de exceso de calor. Asimismo se ha comprobado la existencia de una estrecha relación entre las tipologías de usos del suelo y estructuras urbanas definidas en el Urban Atlas, y los índices de exceso de calor nocturno.
\end{abstract}

Palabras clave: Noche calurosa, estrés térmico, isla de calor, UrbClim, Madrid. 


\title{
Intensity and duration of summer thermal stress in the urban area of Madrid
}

\begin{abstract}
This paper applies a new methodology to the study of hot nights, also known as "tropical" nights, in the metropolitan area of Madrid, in order to evaluate from a temporal and spatial perspective those nights in which the population may be affected by heat stress. The use of two indicators obtained through hourly data, together with the climatic information provided by the UrbClim model, has made it possible to know on a detailed scale the thermal characteristics of the nights of July between 2008 and 2017, thus being able to assess more accurately the risk to the wellbeing and health of the population. The results show a large inter-urban variability in the intensity and duration of heat stress, as well as a significant correlation between heat island intensities and excess heat indices. A close relationship between land use typologies and urban structures as defined in the Urban Atlas and night-time excess heat indices was also found.
\end{abstract}

Keywords: Hot night, heat stress, heat island, UrbClim, Madrid.

\section{Introducción}

Los episodios de calor constituyen un fenómeno recurrente en los climas templados durante el periodo estival, especialmente en las regiones más meridionales y más expuestas a las masas de aire cálidas procedentes de latitudes subtropicales, causantes de dichos episodios. Se trata de un fenómeno que repercute negativamente tanto en el bienestar como en la salud de la población. Los impactos del exceso de calor en la población han sido descritos por diferentes autores, que han encontrado relaciones entre las altas temperaturas y la mortalidad-morbilidad (Bobb et al., 2014; Gronlund et al., 2014; Gasparrini et al., 2015; Guo et al. 2017, Vicedo-Cabrera et al. 2018; Campbell et al., 2018). El último informe del Grupo de Trabajo II del IPCC (2014) señala que hay evidencia de mayor mortalidad relacionada con el calor y menor con el frío en algunas zonas del planeta como Europa, y se advierte del muy probable incremento de la frecuencia e intensidad de los episodios de calor extremo que conllevará el calentamiento global. El IPCC destaca también como muy probable que los días y noches cálidos sean más numerosos y más cálidos, y no siempre asociados a las olas de calor. Diversos estudios muestran relaciones significativas entre un mayor riesgo de mortalidad y una menor amplitud térmica debida a elevados valores de las temperaturas mínimas (Lim et al., 2012; Luo et al., 2013). En dichos casos el estrés térmico persiste y se agrava debido a que se impide al organismo un descanso nocturno de las altas temperaturas diurnas.

La consecuencia más habitual de las noches calurosas sobre la salud es su impacto en el sueño y en el descanso de las personas. El calor puede llevar, entre otros efectos, 
a alteraciones y privaciones de éste debidos a la puesta en marcha de los procesos de termorregulación (Buguet, 2007; Joshi et al., 2016). Concretamente, las temperaturas superiores a las del confort pueden influir en el aumento del desvelo y en la disminución de las fases REM (Rapid Eye Movement) y SWS (Slow-wave Sleep) (Haskella et al., 1981; Okamoto y Mizuno 2005, 2012). Nastos and Matzarakis (2008) encontraron una relación positiva entre la temperatura mínima del aire y las perturbaciones del sueño. Fujii et al. (2015) destacan que las altas temperaturas estivales incrementan la fatiga en la salud. Además, la duración y la calidad del periodo de sueño nocturno son considerados como un factor de riesgo para las personas con problemas cardiovasculares (Nagai et al., 2010; Cappuccio, et al., 2011). Estas alteraciones del sueño se dan con mayor incidencia, al igual que el riesgo general de morbi-mortalidad durante los episodios de calor, en personas con avanzada edad (Buguet, 2007; Koppe et al., 2004).

Es en las ciudades donde los impactos negativos del calor sobre el confort y la salud son mayores, dado que se ven agravados por el fenómeno urbano de "isla de calor". Temperaturas más elevadas que en el entorno, favorecidas por diversos factores urbanos, y el más lento enfriamiento nocturno, generan durante los episodios de calor unas condiciones de mayor estrés térmico y el aumento del riesgo para la salud de los habitantes de las ciudades, en contraste con el medio rural circundante (Moreno 1999; Andrade y Alcoforado, 2008; Laaidi et al., 2012; Royé y Martí, 2015).

Otro factor a tener muy en cuenta es el considerable aumento de los episodios de calor que se ha podido observar a escala global en las últimas décadas (Coumou y Robinson, 2013; Coumou y Rahmstorf, 2012). Según la OMS (Martínez et al., 2019) la población expuesta a las olas de calor está aumentando en grandes regiones del planeta. Arkadiusz et al. (2017) mostraron un significativo incremento de los días calurosos y de las olas de calor en los países del sur y suroeste de Europa entre 1950 y 2006. En la Península Ibérica estimaron que se produjo un aumento de los días calurosos en torno al 0,3\%-1,6\% días por década, hecho también constatado para Madrid por Fernández García y Allende (2016).

Teniendo en cuenta las proyecciones de cambio climático, en las que se estima una mayor frecuencia, duración e intensidad de las olas de calor que afectarán a Europa, con mayores impactos en la Península Ibérica y en las regiones del Mediterráneo (Fischer y Schär 2010; Olcina y Martín 2012; Donat et al. 2013; IPCC 2014; Schleussner et al. 2017; Gasparrini et al. 2017; King y Karoly 2017; Guerreiro et al. 2018, Sousa et al., 2019), consideramos de interés poder disponer de indicadores adecuados que permitan evaluar el riesgo de impacto del calor nocturno sobre la salud y el bienestar de la población, y muy especialmente en las zonas urbanas, donde el fenómeno de isla de calor incrementa ostensiblemente los valores de temperatura alcanzados.

En trabajos recientes se ha podido comprobar que el uso exclusivo de la temperatura mínima como referencia para delimitar las noches tropicales, puede resultar insu- 
ficiente para llevar a cabo un análisis detallado del impacto del calor nocturno en la salud (Royé y Martí, 2015 y 2016; Royé, 2015; Royé y Fernández García, 2017). Por una parte, hay que tener en cuenta que la temperatura mínima suele alcanzarse habitualmente en los momentos próximos a la salida del sol; pero se pueden registrar temperaturas superiores a $20^{\circ} \mathrm{C}$ durante muchas horas de la noche y obtener, como registro mínimo, una temperatura inferior a los $20^{\circ} \mathrm{C}$. En estos casos el estrés térmico no es necesariamente menor que en los días con una temperatura mínima igual o superior a $20^{\circ} \mathrm{C}$, sobre todo si tenemos en cuenta que la fase inicial del sueño, en comparación con las siguientes, se describe como la más sensible y la que acumula las mayores alteraciones por estrés térmico (Okamoto et al., 2005; Okamoto-Mizuno y Mizuno, 2012).

Estas limitaciones llevaron a los autores Royé y Martí $(2015,2016)$ a desarrollar y proponer nuevos indicadores que permitiera cuantificar el exceso y la duración del estrés térmico durante las noches calurosas, lo que mejora la evaluación de este tipo de riesgo térmico. Los índices fueron modificados posteriormente (Royé 2017, Royé et al. 2021) en estudios de cuantificación del riesgo de mortalidad aplicado en el sur de Europa. Se encontró una asociación positiva entre el riesgo relativo de mortalidad por causa específica y ambos índices con una relación convexa no lineal en la mayoría de las ciudades; cuanto mayor es la duración o el exceso, mayor es el riesgo de mortalidad asociada.

Son ya diversos los trabajos que constatan el incremento del estrés térmico en la ciudad de Madrid debido al fenómeno de la isla de calor y de los episodios de calor (Fernández García y Rasilla, 2013; Fernández García y Allende, 2016; Royé y Fernández García, 2017; Xiao et al., 2018; Rasilla et al.,2019; Sanchez-Gevara et al., 2019), así como los efectos del exceso de calor en la salud en esta área urbana (Bardon et al., 2019; García-Lledó et al., 2020).

Con este estudio se pretende, en primer lugar, obtener una aproximación al patrón espacial de exposición al calor dentro de la ciudad y en su periferia, pudiendo así identificar las zonas más frescas y las más expuestas y afectadas por el calor intenso. Y, en segundo lugar, evaluar la relación entre el índice de exceso de calor, el fenómeno de isla de calor y los usos del suelo, lo que permitirá conocer mejor los factores locales que influyen en el clima de esta gran área urbana.

\section{Metodología}

\section{1. Área de estudio}

El área de estudio comprende el área metropolitana de Madrid. Se trata de una zona geográficamente ubicada en el centro de España que ha sido muy transformada por la acción antrópica, con una población de 5.012.504 habitantes (Padrón Municipal, INE, 
2020) en un radio de $50 \mathrm{~km}$ alrededor de Madrid, la ciudad más grande de España con una población de 3.334.730 habitantes. Según los criterios de clasificación climática de Köppen, el clima de Madrid se puede clasificar generalmente como un tipo Csa (mediterráneo continental de verano cálido) con fuertes contrastes térmicos estacionales. El alto porcentaje de anticiclones, la fuerte insolación y la escasez de lluvias son factores que contribuyen a la formación de un clima urbano claramente diferenciado (Fernández García y Martilli, 2011).

\subsection{Datos de temperatura}

Son numerosos los trabajos sobre la isla de calor y los campos térmicos en Madrid (López Gómez et al., 1984 y 1995; Fernández García et al., 1996; Fernández García y Rasilla, 2013; Fernández García y Allende, 2016) realizados a partir de los datos procedentes de estaciones meteorológicas fijas, de la información obtenida a través de aparatos de medición portátiles y la realización de transectos urbanos, o bien de la utilización de imágenes del infrarrojo térmico registradas desde satélite o aviones. Más recientemente disponemos de datos reanálisis de alta resolución para el análisis de la variación espacial de las temperaturas en 100 ciudades europeas. Se trata de datos generados por el modelo climático UrbClim (De Ridder et al., 2015) que permite estimar y analizar el efecto isla de calor urbano y otras variables climáticas con una resolución espacial de $100 \mathrm{~m}$ y resolución temporal horaria.

El conjunto de datos contiene la temperatura del aire, la humedad relativa y la velocidad del viento. Estos datos se generaron utilizando el modelo UrbClim, desarrollado en VITO para el Servicio de Cambio Climático de Copernicus. El modelo requiere dos tipos de datos de entrada: datos meteorológicos a gran escala (obtenidos a través de reanálisis ERA5), y una descripción del terreno en la ciudad (uso del suelo, \% suelo edificado, vegetación, elevación, flujo de calor antrópico). Las intensidades de la isla de calor se han estimado usando la diferencia entre el percentil $90 \%$ y el 10\% a las 02:00 h, lo que permite obtener una referencia de zonas con bajas temperaturas (rural) y con altas temperaturas (urbana) evitando valores atípicos (Martin-Vide et al. 2015).

Los datos de temperaturas máximas y mínimas registradas durante la ola de calor de julio de 2017 en varios observatorios españoles se han obtenido de la base de datos de AEMET.

\subsection{Datos de uso de suelo urbano}

Para poder explicar los patrones espaciales relacionados con el exceso de calor nocturno en Madrid, usamos el atlas urbano 2018 con una resolución espacial de 10m (European Environment Agency/Copernicus Land Monitoring Service). 


\subsection{Indices de exceso y duración del calor nocturno}

Los índices de noches cálidas propuestos por Royé (2017, 2021) se basan en datos de temperatura del aire a escala (sub)horaria. La duración de la noche calurosa HNd (Hot Night duration), que describe la duración del efecto de calor, se calcula como la suma de las horas durante la noche durante las cuales se supera un umbral de temperatura $\left(\mathrm{T}_{t b r}\right)$ (el percentil del $95 \%$ de la temperatura mínima).

$$
H N d_{j}=\sum_{j=1}^{n_{j}} \mathrm{I}_{T h h r}\left(t_{i j}\right)
$$

dónde: $n_{i}$ es el número de días horas del día j, $t_{i j}$ : temperatura media durante la hora $i$ en la noche $j, \mathrm{y} \mathrm{I}_{T t h r}$ es la función de índice de $\left\{x \in \mathbb{R} \mid x>\mathrm{T}_{t h r}\right\}$, es decir:

$$
\mathrm{I}_{T t h r}\left(t_{i j}\right)=\left\{\begin{array}{l}
0 \text { if } t_{i j}<T t h r \\
1 \text { if } t_{i j} \geq T t h r
\end{array}\right.
$$

En un segundo índice, la intensidad de las noches calurosas (HNe), permite evaluar la intensidad del estrés térmico nocturno, se obtiene mediante la suma del exceso de calor durante el período de tiempo con temperaturas iguales o superiores al umbral establecido.

$$
H N e_{j}=\sum_{j=1}^{n_{j}}\left(t_{i j}-T t h r\right) \cdot \mathrm{I}_{T t h r}\left(t_{i j}\right)
$$

\section{Resultados y discusión}

\subsection{Isla de calor}

Durante las noches de julio entre 2008 y 2017 la isla de calor de Madrid alcanzó intensidades medias comprendidas entre los $4^{\circ} \mathrm{C}$ y $\operatorname{los} 6^{\circ} \mathrm{C}$ (Figura 1 ), valores similares a los obtenidos en anteriores estudios para los meses de verano (Fernández y Allende, 2016, Xiao et al. 2017, Sobrino et al. 2012, Sanchez-Guevara et al. 2019, Rasilla et al. 2019). Dependiendo de las situaciones atmosféricas diarias las intensidades de la isla de calor han registrado un rango mayor de variación, con valores que bien han llegado a superar puntualmente $\operatorname{los} 8^{\circ} \mathrm{C}$ de diferencia entre el centro de la ciudad y algunas áreas de su periferia, o, por el contrario, han quedado por debajo de los $2^{\circ} \mathrm{C}$ de variación.

En la madrugada del 31 de julio de 2013 se observó la isla de calor de mayor intensidad en el periodo analizado, con una variación máxima de 9․․ La situación atmosférica estuvo condicionada por el anticiclón de las Azores, que en forma de cuña abarcaba toda la Península Ibérica bajo su radio de acción, generando una situación de fuerte estabilidad. El día 30 la insolación había sido muy alta (13,5h). La temperatura máxima 
en el observatorio del Retiro alcanzó los 33,5으, llegando a 37으 el día 31. Las temperaturas mínimas registradas en la madrugada del día 31 oscilaron desde los $16^{\circ} \mathrm{C}$ de Torrejón, pasando por los $21^{\circ} \mathrm{C}$ del observatorio del Retiro, hasta los $22^{\circ} \mathrm{C}$ de Getafe, al sur de la ciudad. La configuración de la isla de calor mostraba un desplazamiento del centro de ésta hacia el suroeste, posiblemente debido a la acción del viento que soplaba desde el $\mathrm{N}$ y NE con velocidades medias entre los $2,2 \mathrm{~m} / \mathrm{s}$ del Retiro y los 4,4 m/s en Torrejón y Barajas (Figura 2). Con esta situación fueron los barrios al suroeste de Madrid, así como los núcleos de población de Leganés, Getafe o Fuenlabrada, los que registraron a primeras horas de la noche las temperaturas más elevadas, por encima de los $28^{\circ} \mathrm{C}$. En el centro de la ciudad las temperaturas fueron más bajas, entre $25^{\circ} \mathrm{C}$ y $28^{\circ} \mathrm{C}$, mientras que los sectores situados al noreste, norte y noroeste (Pozuelo, Alcobendas, Barajas, Torrejón, La Moraleja o Coslada) disfrutaron de un ambiente más suave y fresco, con valores entre $19^{\circ} \mathrm{C}$ y $24^{\circ} \mathrm{C}$. Fernández García et al. (2016) identificaron un tipo de comportamiento en días extremadamente calurosos en los que el calor fue más intenso en algunas de las zonas suburbanas que en las zonas urbanas, lo que podría tener cierta relación con situaciones de este tipo, en las que el viento desplaza el centro de la isla de calor hacia algunos de los núcleos urbanos de la periferia. Otra posibilidad, ya señalada por Fernández et al. (1996), estaría relacionada con la entrada de aire fresco procedente de zonas extraurbanas, especialmente del piedemonte de las sierras al norte y noreste de Madrid, canalizado por los valles del Manzanares y del Jarama.

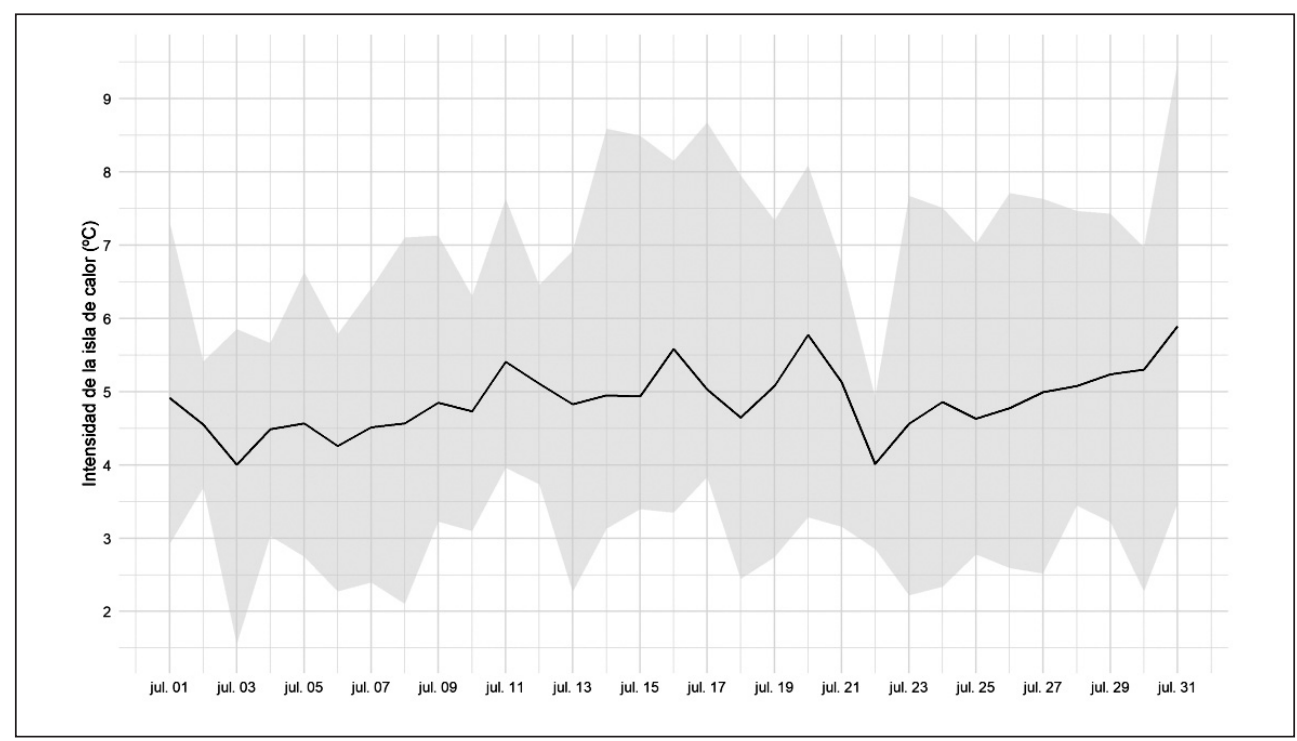

Figura 1. Intensidades medias y extremas de la isla de calor de Madrid en el mes de julio (2008-2017). 
Una situación atmosférica muy diferente caracterizó el día 3 de julio de 2014, cuando una baja térmica en superficie, junto a una línea de inestabilidad que atravesaba la Península de este a oeste, coincidieron con un embolsamiento de aire frío en altura, lo que generó una situación de fuerte inestabilidad. Ese día se registraron 1,5 mm de precipitación en el Retiro, una insolación de solo 4,4 h y una temperatura máxima de $22,9^{\circ} \mathrm{C}$. Hubo una diferencia de $1,5^{\circ} \mathrm{C}$ entre el sector con la temperatura más alta $\left(16,8^{\circ} \mathrm{C}\right)$ y el que registraba la temperatura más baja $\left(15,3^{\circ} \mathrm{C}\right)$. Fue la isla de calor más débil registrada en el mes de julio entre 2008 y 2017, condicionada por una atmósfera muy inestable y con importantes turbulencias asociadas a los episodios de tormenta que mezclaron las masas de aire en toda el área metropolitana de Madrid.

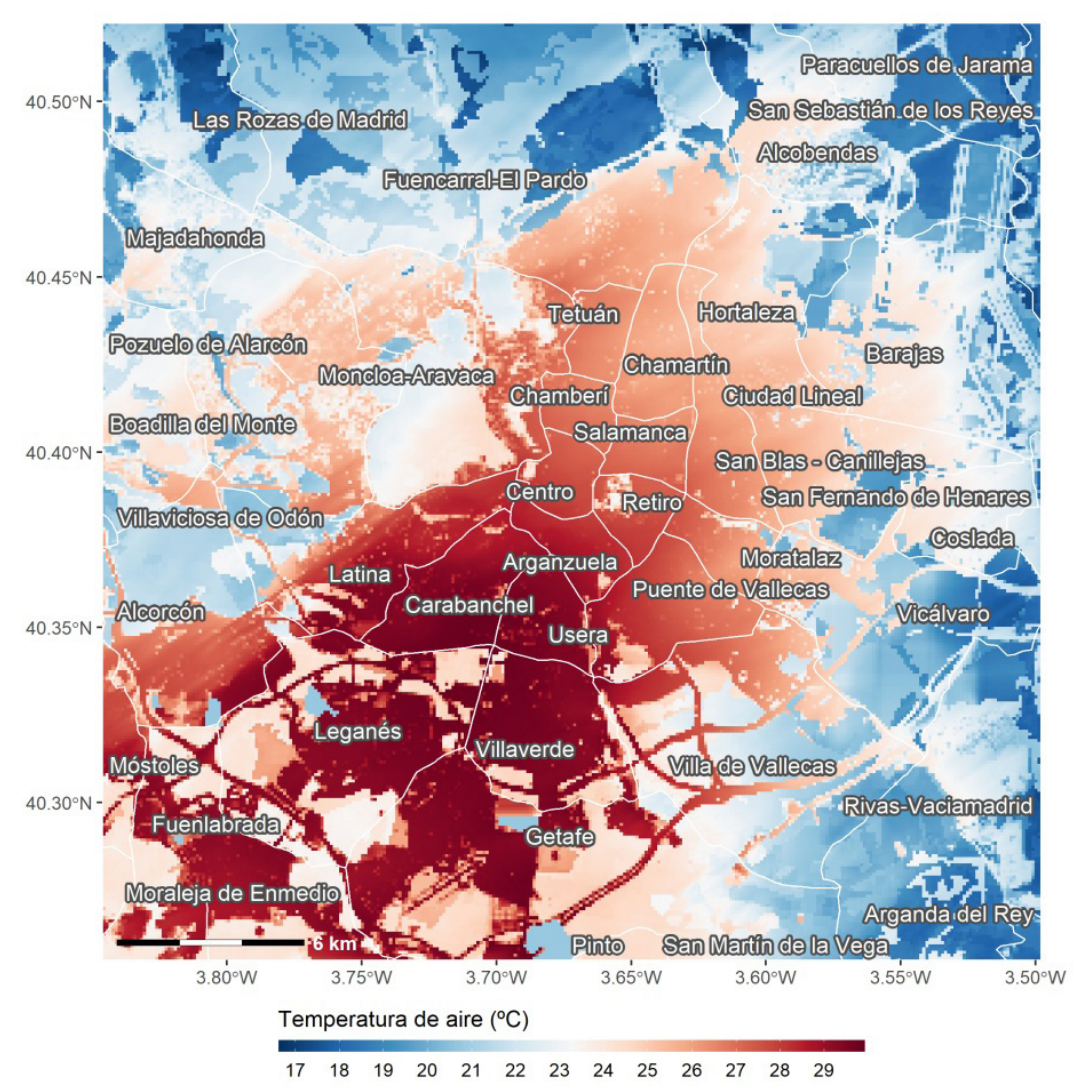

Figura 2. Isla de calor de Madrid en la noche del 31 de julio de 2013.

Fuente: UrbClim, Servicio de Cambio Climático, Copernicus. 


\subsection{Exceso de calor}

Los HNe obtenidos para el mes de julio muestran como, en promedio, durante todas las noches se producen situaciones de estrés térmico en el conjunto del área urbana y periurbana de Madrid, con valores de calor acumulado comprendidos entre $30^{\circ} \mathrm{C}$ y $45^{\circ} \mathrm{C}$ (Figura 3). Pero como en el caso de la intensidad de la isla de calor, dependiendo de las condiciones atmosféricas, estos valores han sido mucho mayores, habiéndose registrado en determinados días índices de exceso de calor superiores a $90^{\circ} \mathrm{C}$. La noche del 18/19 de julio de 2016 se alcanzó el valor máximo de la serie analizada, habiéndose acumulado un total de $124^{\circ} \mathrm{C}$. Este intenso episodio de estrés térmico fue provocado por una situación atmosférica que favoreció la advección hasta el centro peninsular de una masa de aire muy cálida procedente del norte de África. Las temperaturas mínimas en la madrugada del 19 estuvieron comprendidas entre los 21,70ㄷ de Torrejón y los $28,5^{\circ} \mathrm{C}$ de Getafe. Se observa asimismo una suave tendencia a que los excesos de calor acumulados sean mayores conforme avanza el mes de julio, tendencia paralela al constante incremento que sufren las temperaturas diarias estivales hasta comienzos del mes de agosto en que empiezan a revertir.

Se ha correlacionado la UHI y NHe de julio entre 2008 a 2017 (Figura 4). Entre ambas variables existe una correlación significativa de 0.34 (IC95\%, 0.24-0.44, p < 0.001), resultado análogo al obtenido por Fernández García et al. (2016), que constataron que la intensidad de la isla de calor en Madrid aumenta en los periodos cálidos y durante las olas de calor. Royé y Fernández (2017) también advirtieron que existe una relación positiva entre los días con ola de calor y la duración de las noches cálidas. En la correlación entre HNe y UHI se observa como las islas de calor débiles, normalmente debidas a situaciones atmosféricas de mayor inestabilidad o con vientos más intensos, se corresponden con un menor estrés térmico por excesos de calor. Las islas de carácter moderado, entre los $3^{\circ} \mathrm{C}$ y los $6^{\circ} \mathrm{C}$ (Fernández García y Allende, 2016), pueden formarse con masas de aire de características térmicas muy distintas, más suaves o más cálidas, por lo que los HNe son muy variados y presentan una gran dispersión estadística. Finalmente, las islas de calor fuertes y muy fuertes, con intensidades comprendidas entre $4^{\underline{O}}$ y $8^{\circ} \mathrm{C}$ se corresponden habitualmente con noches calurosas que provocan excesos de calor moderados o altos.

Durante el verano de 2017 se registraron en la Península Ibérica cinco olas de calor. La segunda ola de calor, con una duración de 5 días, se registró entre el 12 y el 16 de julio, y afectó a 14 provincias el día 14 de julio, generada por la llegada de una masa de aire muy cálida desde el norte de África. El día más cálido fue el 13, cuando en el centro y sur peninsular se superaron ampliamente los $40^{\circ} \mathrm{C}$, dándose los valores más altos en los valles del Guadiana y del Guadalquivir con temperaturas por encima de $45^{\circ} \mathrm{C}$. Se registraron numerosas efemérides de temperatura máxima absoluta, todas ellas el día 13, entre las que cabe mencionar Córdoba/Aeropuerto con 46,9드. Granada/Aeropuerto con $45,7^{\circ} \mathrm{C}$, Badajoz/Talavera la Real con $45,4 \stackrel{\circ}{\circ}$, Cáceres con $43,2^{\circ} \mathrm{C}$ o To- 


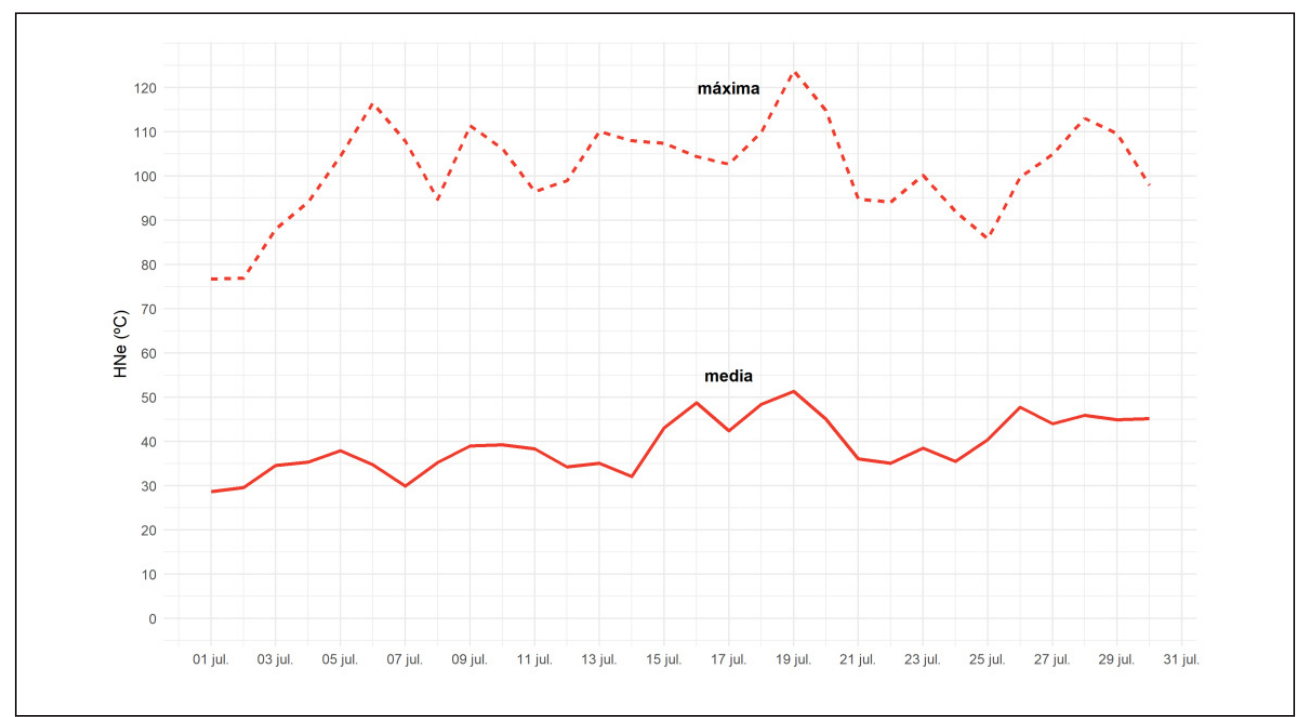

Figura 3. Índices de exceso de calor medios y máximos en el mes de julio (2008-2017). Fuente: UrbClim, Servicio de Cambio Climático, Copernicus.

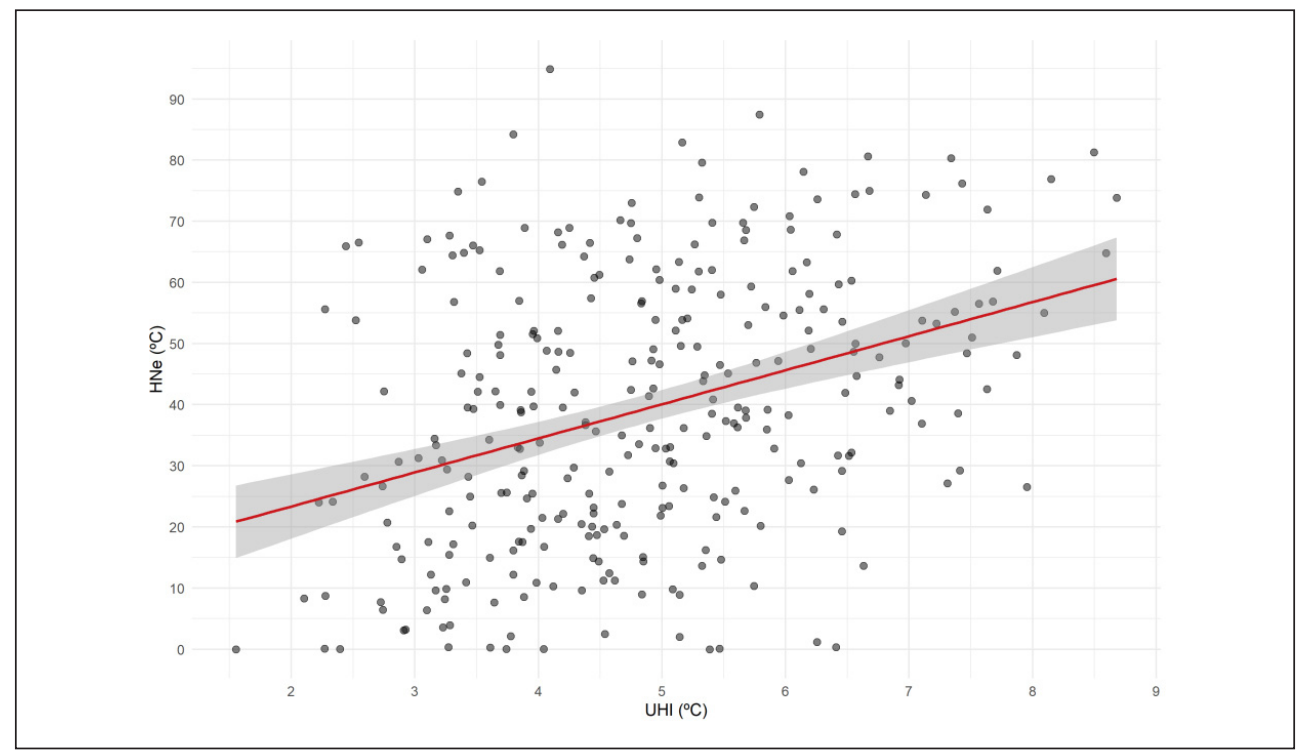

Figura 4. Relación entre los índices de exceso de calor y las intensidades de la isla de calor en Madrid. Fuente: UrbClim, Servicio de Cambio Climático, Copernicus. 
ledo con $42,8^{\circ} \mathrm{C}$. En Madrid, en el observatorio de Cuatro Vientos se registraron $40,6^{\circ} \mathrm{C}$, y en el Retiro $39,7^{\circ} \mathrm{C}$. También las mínimas fueron muy elevadas, con valores próximos a $30^{\circ} \mathrm{C}$ en puntos del interior de Andalucía. Entre las temperaturas mínimas alcanzadas estos días en las estaciones principales de AEMET destacan los $28,5^{\circ} \mathrm{C}$ de Jaén el día 13. En el área metropolitana de Madrid se registraron $25,4^{\circ} \mathrm{C}$ en Getafe el día 14, $24,7^{\circ} \mathrm{C}$ en el Retiro, 24, $6^{\circ} \mathrm{C}$ en Cuatro Vientos, $21,6^{\circ} \mathrm{C}$ en Barajas, $21,4^{\circ} \mathrm{C}$ en Torrejón y $23,2^{\circ} \mathrm{C}$ en Colmenar Viejo. Esa misma madrugada del día 14 la isla de calor de Madrid alcanzó una intensidad próxima a los $7^{\circ} \mathrm{C}$.

Durante la noche del día 9/10, y tras unos días de inestabilidad atmosférica con episodios de tormenta en el centro peninsular, los excesos de calor fueron en general débiles. Es a partir de la noche del día 11 cuando las temperaturas sufrieron un considerable aumento que se notó especialmente en los valores nocturnos y de madrugada, con mínimas que no descendieron de los $20^{\circ} \mathrm{C}$ en el centro de Madrid y en las áreas urbanas del suroeste (Figura 5). La noche del 12 al 13 fue ya extremadamente calurosa; los índices HNe superaron los $80^{\circ} \mathrm{C}$ en todo en todo el centro urbano de Madrid, especialmente en algunos sectores del noreste. Tras un día de calor extremo con máximas el día 13 en torno a los $40^{\circ} \mathrm{C}$, los excesos de calor superaron esa noche los $90^{\circ} \mathrm{C}$ acumulados en buena parte del área urbana de Madrid y de los núcleos de población del suroeste, como Leganés, Getafe o Fuenlabrada. En los sectores del norte de la ciudad (Valverde, Tetuán, Alcobendas, la Moraleja o Pozuelo) los valores de HNe fueron algo más bajos, entre $50^{\circ} \mathrm{C}$ y $90^{\circ} \mathrm{C}$. En las siguientes noches el estrés térmico fue menor, pero todavía con valores de HNe comprendidos entre $70^{\circ} \mathrm{C}$ y $90^{\circ} \mathrm{C}$ en la mayor parte del área urbana y periurbana. Es a partir del día 19 cuando la ola de calor remite y las noches de calor y el estrés térmico se suavizan ostensiblemente, con índices de exceso de calor ya por debajo de $\operatorname{los} 50^{\circ} \mathrm{C}$.

Las diferencias interurbanas se observan más claramente en el mapa de la Figura 6, donde se sintetiza el comportamiento del conjunto de las noches calurosas del mes de julio de 2017 en el área metropolitana de Madrid, teniendo en cuenta tanto el exceso de calor (HNe), como el número de horas durante la noche en las que la temperatura umbral $\left(22,5^{\circ} \mathrm{C}\right)$ fue excedida $(\mathrm{HNd})$. El resultado de la interacción entre ambas variables, intensidad y duración, constituye una mejor aproximación al estrés térmico nocturno que sufrieron los habitantes de la ciudad y de su entorno.

Todo el centro urbano y algunos de los núcleos de población próximos al sur y este (Leganés, Getafe, Rivas, Coslada y núcleo antiguo de Barajas) registraron excesos de calor moderados además de una elevada duración del estrés térmico durante las noches de julio, que alcanzaron el 100\% de la noche durante el episodio de ola de calor descrito. Los núcleos urbanos al oeste y al norte, desde Fuenlabrada y Alcorcón, hasta San Sebastián de los Reyes, se caracterizaron durante ese mes de julio por unos valores medios tanto de la intensidad del calor como de su duración. Y fueron las áreas no urbanizadas de la periferia, como los montes del Pardo al noroeste, o el valle del 


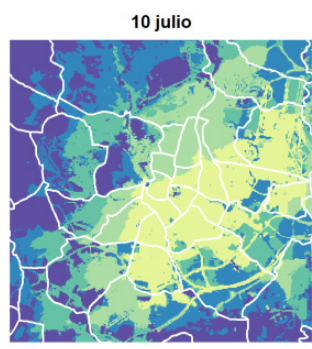

14 julio

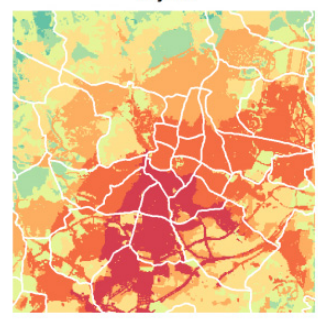

18 julio

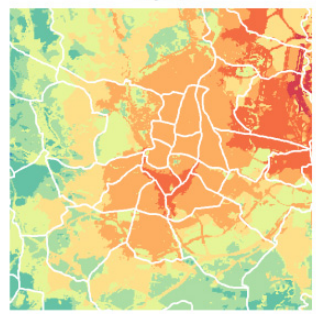

12 julio

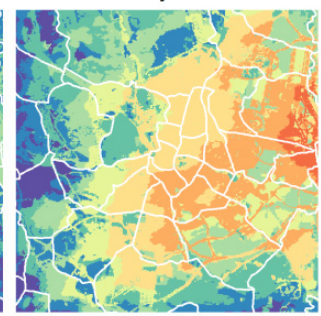

15 julio

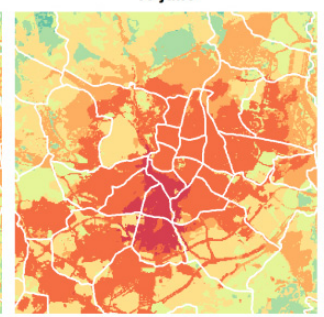

19 julio

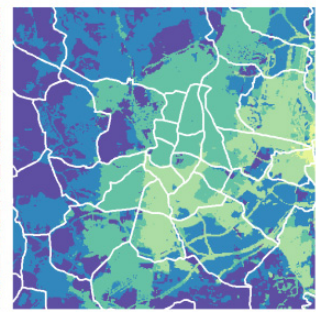

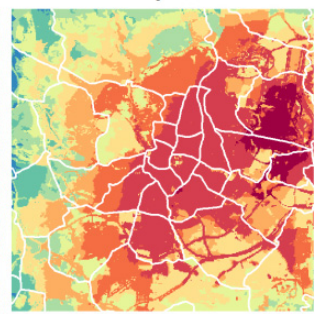

16 julio

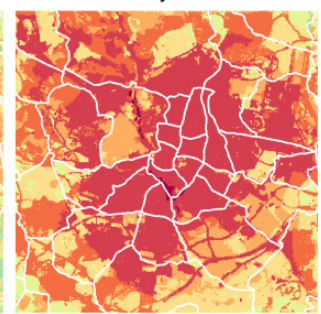

20 julio

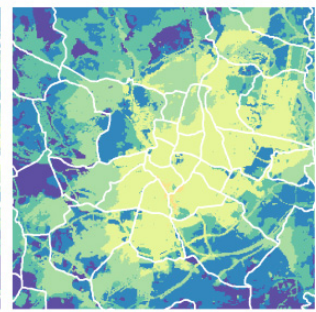

13 julio

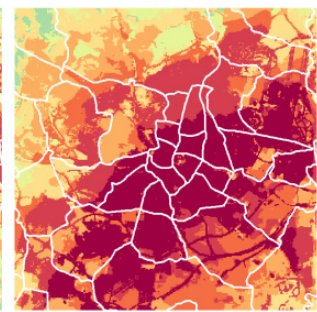

17 julio

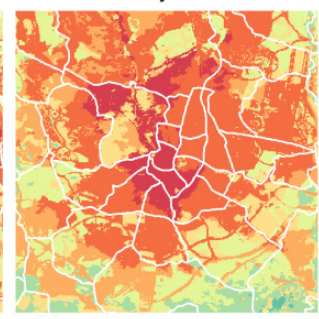

21 julio

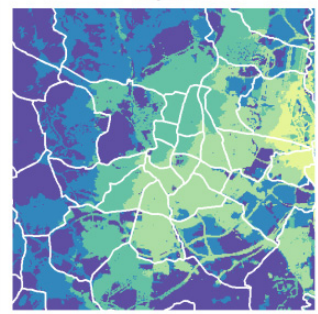

$\mathrm{HNe}\left({ }^{\circ} \mathrm{C}\right)$

$\begin{array}{lllllllll}17.7 & 27.2 & 36.7 & 45.4 & 54.0 & 63.2 & 72.5 & 81.4 & 92.6\end{array}$

Figura 5. Índices de exceso de calor durante la ola de calor de julio de 2017.

Fuente: UrbClim, Servicio de Cambio Climático, Copernicus.

río Manzanares al sur, junto con algunos sectores urbanos como Torrejón o el aeropuerto, así como los grandes espacios verdes en el área urbana (Casa de Campo, Parque del Retiro) o periurbana (Parque forestal Felipe VI), los que se beneficiaron de un menor estrés térmico nocturno, con unos índices de exceso de calor de bajos a moderados, y una menor persistencia de los altas temperaturas.

Todos los estudios realizados sobre la isla de calor coinciden en que los usos del suelo y las estructuras urbanas son los principales factores explicativos del clima urbano y de las diferencias que se observan en el interior de la ciudad. Por ello se han relacionado los índices diarios de exceso de calor nocturno del mes de julio 2017 con las tipologías de usos del suelo y estructuras urbanas obtenidas del URBAN ATLAS. Los menores índices de exceso de calor $\left(<40^{\circ} \mathrm{C}\right.$ acumulados) están asociados a espacios abiertos, zO- 


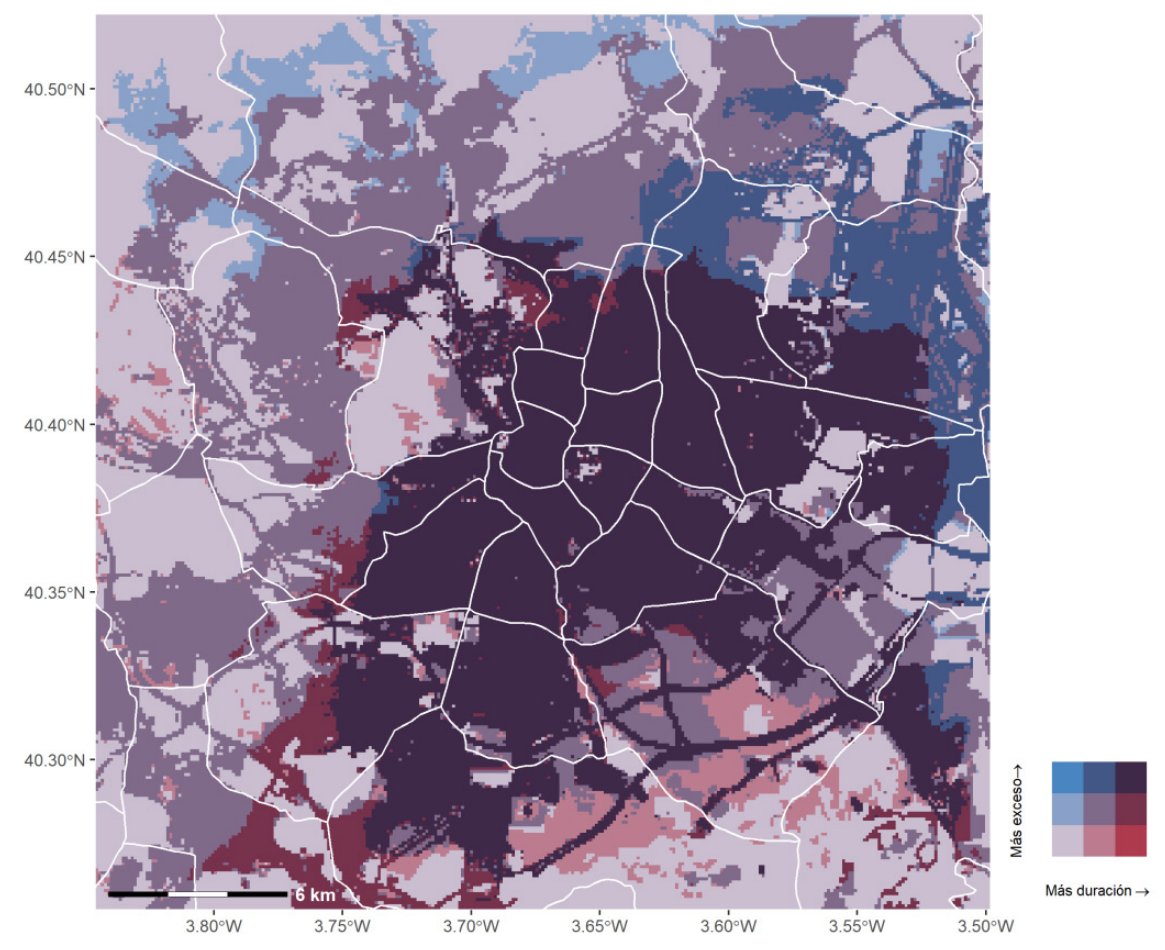

Figura 6. Intensidad y duración del estrés térmico en el conjunto de las noches calurosas del mes de julio de 2017.

Fuente: UrbClim, Servicio de Cambio Climático, Copernicus.

nas húmedas y láminas de agua, campos de cultivo, pastos, bosques o áreas urbanas de muy baja densidad ( $<10 \%$ suelo urbanizado), donde, además, son predominantes los espacios y elementos verdes (Figura 7). Conforme la densidad constructiva y el porcentaje de suelo urbanizado aumentan, los valores promedio y extremos del exceso de calor y del estrés térmico nocturno se incrementan. Dichos índices alcanzan valores promedio próximos a $70^{\circ} \mathrm{C}$ en aquellos sectores con $>50 \%$ de suelo urbanizado, en estaciones de tren o en calles con una elevada densidad de tráfico. Fernández y Allende (2016) obtuvieron resultados similares, con correlaciones positivas y significativas con el índice de compacidad, el área construida densa y con el área impermeable. La correlación fue negativa con la superficie permeable y las áreas agrícolas y forestales. 


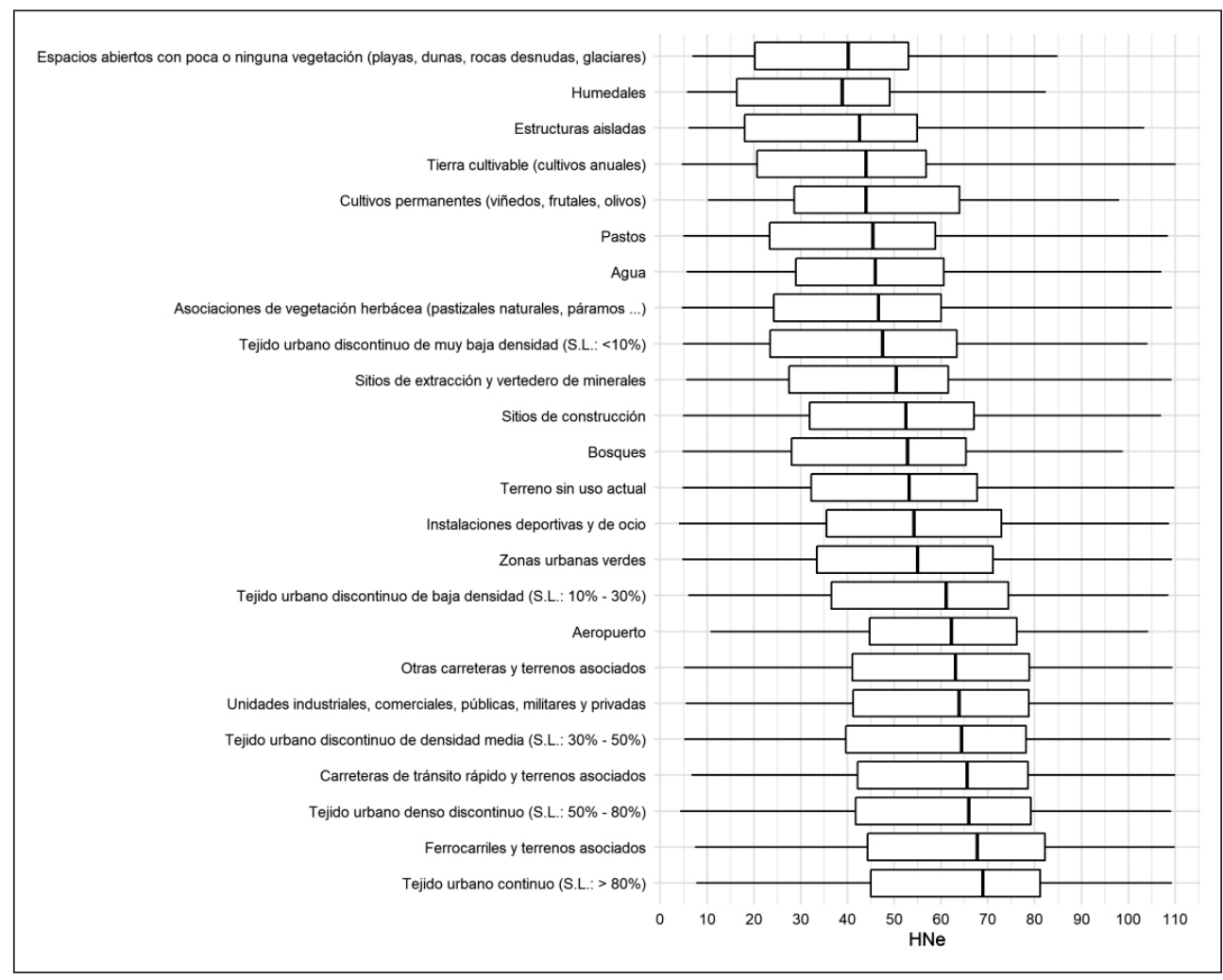

Figura 7. Índices de exceso de calor según los usos del suelo en el área urbana de Madrid durante durante las noches calurosas del mes de julio de 2017.

Fuente: Atlas Urbano de 2018, European Environment Agency/Copernicus Land Monitoring Service.

\section{Conclusiones}

El incremento de los episodios de calor en las últimas décadas es un hecho científicamente demostrado. Los escenarios de futuro indican claramente que los riesgos para la salud por calor se seguirán incrementando, especialmente de los espacios urbanos. La OMS advierte que la población expuesta a las olas de calor está aumentando, e insiste en la necesidad de adaptación a este tipo de riesgo a través de planes de acción, desarrollando planes de alerta y comunicación, estudios sobre los cambios en la sensibilidad al calor de la población, o mejoras en la práctica de la gobernanza y en el diseño urbano respetuoso con el clima. Los impactos parecen disminuir con medidas preventivas y de adaptación sanitaria, pero necesitan ser modificadas para ser efectivas en las próximas décadas con el cambio climático. 
Entre dichas medidas está el uso de nuevas fuentes de información e indicadores que permitan conocer con más precisión el grado de riesgo por calor que soporta la población. El método propuesto en este trabajo para la caracterización de las noches cálidas, basado en el uso de datos horarios procedentes del modelo climático UrbClim, junto a los índices de intensidad y duración del exceso de calor ha permitido realizar una aproximación detallada del ambiente térmico nocturno en el área metropolitana de Madrid en verano, así como del riesgo para el bienestar y la salud de su población. En anteriores estudios realizados en distintos ámbitos de la Península Ibérica, ya pudo comprobarse como estos índices permiten calcular con más precisión el número de noches con posible estrés térmico, que en numerosas ocasiones quedan ocultas al utilizar sólo los valores de las temperaturas mínimas.

La aplicación de esta metodología al área de estudio muestra, en primer lugar, una fuerte variabilidad temporal de la intensidad de la isla de calor urbana, así como del índice de exceso de calor nocturno en función de las situaciones atmosféricas durante el mes de julio en el periodo 2008-2017. Los índices reflejan también una gran variación climática interurbana. El centro de la ciudad, junto a los núcleos de población al suroeste de la misma son los sectores que padecen con mayor frecuencia e intensidad las situaciones de estrés térmico nocturno. En el otro extremo se encuentran los barrios y núcleos de población situados más al norte, noreste y noroeste, favorecidos por el efecto suavizador de las masas de aire más frescas procedentes de los espacios de montaña próximos y de la entrada de flujos más frescos canalizados por los valles de los ríos Manzanares y Jarama.

Existe una correlación significativa moderada entre la intensidad de la isla de calor y el exceso de calor nocturno Los mayores índices de exceso de calor coinciden habitualmente con las islas de calor de mayor intensidad, con fuertes contrastes entre el centro y suroeste de la ciudad, y los sectores más septentrionales.

Finalmente se ha comprobado la existencia de una relación entre las tipologías de usos del suelo y estructuras urbanas definidas en el Urban Atlas, y los índices diarios de exceso de calor nocturno registrados en el área metropolitana. El índice de compacidad y el porcentaje de área impermeable y construida constituyen importantes potenciadores de la temperatura y del aumento del estrés térmico durante las noches calurosas en Madrid.

\section{Bibliografía}

Andrade, H. y Alcoforado, M.-J. (2008). Microclimatic variation of thermal comfort in a district of Lisbon (Telheiras) at night. Theor. Appl. Climatol., 92, pp. 225-237.

Arkadiusz, M; Tomczyk; Półrolniczak, M; Bednorz, E. (2017). Circulation Conditions' Effect on the Occurrence of HeatWaves in Western and Southwestern Europe. Atmosphere 2017, 8, 31; doi:10.3390/atmos8020031 
Bardón Iglesias R, Cámara Díez E, Rivera Ariza S, Gandarillas Grande A, Ordobás Gavín M, Fúster Lorán F. (2019). Vigilancia y control de los efectos de las olas de calor en la salud en la Comunidad de Madrid. REMASP. 2019; 2(13): pp. 1-3.

Bobb, J.F.; Peng, R.D.; Bell, M.L. y Dominici, F. (2014). Heat-Related Mortality and Adaptation to Heat in the United States. Environmental Health Perspectives, 122: pp. 811-816.

Buguet, A. (2007). Sleep under extreme environments: Effects of heat and cold exposure, altitude, hyperbaric pressure and microgravity in space. Journal of the Neurological Sciences, 262, pp. 145-152.

Campbell S; Remenyi TA; White CJ; Johnston FH (2018). Heatwave and health impact research: a global review. Health \& Place. 53: pp. 210-218. doi:10.1016/j.healthplace.2018.08.017

Cappuccio, FP; Cooper, D; D’Elia, L; Strazzullo, P; Miller, MA (2011): 'Sleep duration predicts cardiovascular outcomes: a systematic review and meta analysis of prospective studies', Eur Heart J, 32: pp. 1484-1492.

Coumou, D. y Rahmstorf, S. (2012). A decade of weather extremes. Nature Climate Change, 2, pp. 491-496.

Coumou, D. y Robinson, A. (2013). Historic and future increase in the global land area affected by monthly heat extremes. Environ. Res. Lett., 8.

De Ridder, K., D. Lauwaet and B. Maiheu (2015). UrbClim - A fast urban boundary layer climate model, Urban Climate, Vol. 12, pp. 21-48. https://doi.org/10.1016/j.uclim.2015.01.001

Donat, M.G., et al. (2013). Updated analyses of temperature and precipitation extreme indices since the beginning of the twentieth century. Journal of Geophysical Research: Atmospheres, 118, pp. 1-16.

Fernández Garcia, F. y Allende, F (dir.) (2016). Estudio de detalle del clima urbano de Madrid. Ayuntamiento de Madrid, 209 p.

Fernández García, F., Rasilla Álvarez, D. (2013). Extreme warm events and urban confort in the metropolitana rea of Madrid: Proposal of an urban heat index (UHINDEX). Two hundred years of urban meteorology (Geogiadis M. A et al. coor), pp. 144-152.

Fernández García, F.; Almendros, M.A.; López Gómez, A. (1996). La influencia del relieve en la isla de calor de Madrid. Estudios Geográficos, Vol 57, no 224, pp. 473-494.

Fernández García, F; Martilli, A (2011): Urban climate research in Spain, International Association for UrbanClimate, 40, pp. 22-28.

Fischer, E.M. y Schär, C. (2010). Consistent geographical patterns of changes in high-impact European heatwaves, Nature Geoscience, 3, pp. 398-403.

Fujii, H; Fukuda, S; Narumi, D; Ihara, T; Watanabe, Y (2015). Fatigue and sleep under large summer temperature differences. Environmental Research, 138: pp. 17-21.

García-Lledó,A; Rodríguez-Martín, S; Tobías, A; Alonso, J; Ansede, JC; de Abajo, FJ. (2020). Heat waves, ambient temperature, and risk of myocardial infarction: an ecological study in the Community of Madrid Revista Española de Cardiología (English Edition), Volume 73, Issue 4, April, Pages 300-306. https://doi.org/10.1016/j.recesp.2019.05.009 
Gasparrini, A.; Guo, Y.; Sera, F.; Vicedo-Cabrera, A.M.; Huber, V.; Tong, S.; Armstrong, B. (2017). Projections of temperature-related excess mortality under climate change scenarios. Lancet Planet. Health 1 e360-7

Gasparrini, A; Guo, Y; Hashizume, M; Lavigne, E; Zanobetti, A; Schwartz, J; Tobias, A; Tong, S; Rocklöv, J;Forsberg, B; Leone, M; De Sario, M; Bell, ML; Guo, YLL; Wu, C; Kan, H; Yi, SM; Coelho, M; Saldiva, P; Honda,Y; Kim, H; Armstrong, B (2015). Mortality risk attributable to high and low ambient temperature: a multicountry observational study, Lancet, 386: pp. 369-375.

Gronlund, C.J.; Zanobetti, A.; Schwartz, J.D.; Wellenius, G.A. and O'Neill, M.S. (2014). Heat, Heat Waves, and Hospital Admissions among the Elderly in the United States, 1992-2006. Environmental Health Perspectives, 122, pp. 1187-1192.

Guerreiro, S.B.; Dawson, R.J.; Kilsby, C.; Lewis, E.; Ford, A. (2018). Future heat waves, droughts and floods in 571 European cities. Environ. Res. Lett., 13(3): 034009

Guo Y, Gasparrini A, Armstrong BG, Tawatsupa B, Tobias A, Lavigne E, Coelho MSZS, Pan X, Kim H, Hashizume M, Honda Y, Guo YL, Wu CF, Zanobetti A, Schwartz JD, Bell ML, Scortichini M, Michelozzi P, Punnasiri K, Li S, Tian L, Garcia SDO, Seposo X, Overcenco A, Zeka A, Goodman P, Dang TN, Dung DV, Mayvaneh F, Saldiva PHN, Williams G, Tong S. (2017). Heat Wave and Mortality: A Multicountry, Multicommunity Study. Environ Health Perspect. Aug 10;125(8):087006. Doi: 10.1289/EHP1026. PMID: 28886602; PMCID: PMC5783630.

Haskella E.H.; Palcaa J.W.; Walkera J.M.; Bergera R.J.; Hellera H.C. (1981). The effects of high and low ambient temperatures on human sleep stages. Electroencephalography and Clinical Neurophysiology, 51, pp. 494-501.

IPCC (2014). Impacts, Adaptation and Vulnerability. Working Group II Contribution to AR5.

Joshi, SS; Lesser, TJ; Olsen, JW; O'Hara, BF (2016). 'The importance of temper ature and thermoregulation for optimal human sleep', Energy and Buildings, 131: pp. 153-157.

King, A.D.; Karoly, D.J. (2017). Climate extremes in Europe at 1.5 and 2 degrees of global warming. Environ. Res. Lett. 12, 114031

Koppe, C.; Kovats, S.; Jendritzky, G. y Menne, B. (2004). Heat-waves: risks and responses, WHO Regional Office for Europe.

Laaidi, K.; Zeghnoun, A.; Dousset, B.; Bretin, P.; Vandentorren, S.; Giraudet, E. y Beaudeau, P. (2012). The Impact of Heat Islands on Mortality in Paris during the August 2003 Heat Wave. Environmental Health Perspectives, 120, pp. 254-259.

Lim, Y.H.; Hong, Y.C.; Kim, H. (2012). 'Effects of diurnal temperature range on cardiovascular and respiratory hospital admissions in Korea', Sci Total Environ, 417-418: pp. 55-60.

López Gómez, A. y Fernández García, F. (1984). La isla de calor en Madrid: avance de un estudio de clima urbano, Estudios Geográficos, n. 174, pp. 5 a 34.

López Gómez, A; López, J; Fernández García, F; Moreno, A. (1995). El clima urbano. Teledetección de la isla de calor en Madrid. Estudios Geográficos; Tomo 56, N. 219): p. 463.

Luo, Y; Zhang, Y; Liu, T; Rutherford, S; Xu, Y; Xu, X; Wu, W; Xiao, J; Zeng, W; Chu, C; Ma, W (2013). Lagge effect of diurnal temperature range on mortality in a subtropical megacity of china, PLOS ONE, 8(2):e55280. 
Martinez, G; Linares, C; Ayuso, A; Kendrovskic,V; Boeckmannd, M. (2019). Heat-health action plans in Europe: Challenges ahead and how to tackle Them. Environmental Research 176. ttps://doi.org/10.1016/j.envres.2019.108548

Martin-Vide, J.; Sarricolea P.; Moreno-García, M.C. (2015). On the definition of urban heat island intensity: the "rural" reference. Front. Earth Sci. 3: p. 24. Doi: 10.3389/feart.2015.00024

Moreno García, M.C.(1999): Climatología urbana. Edicions Universitat de Barcelona. 71 pp.

Nagai, M.; Hoshide, S.; Kario, K. (2010). 'Sleep duration as a risk factor for cardiovascular diseasea review of the recent literature', Curr Cardiol Rev, 6: pp. 54-61.

Nastos P.T. y Matzarakis A. (2008). Human-Biometeorological effects on sleep disturbances in Athens, Greece: A Preliminary Evaluation. Indoor Built Environment, 17, pp. 535-542.

Okamoto-Mizuno K. y Mizuno K. (2012). Effects of thermal environment on sleep and circadian rhythm, Journal of Physiological Anthropology, 31:1:14.

Okamoto-Mizuno, K.; Tsuzuki, K. y Mizuno K. (2005). Effects of humid heat exposure in later sleep segments on sleep stages and body temperature in humans. Int J Biometeorol, 49, pp. 232-237.

Olcina, J., Martin, D. (2012): "Variaciones en la densidad del oxígeno en el aire y su influencia sobre la salud humana". Boletín de la Asociación de Geógrafos Españoles, no ${ }^{\circ}$ 8, pp. 7-32.

Rasilla, D; Allende, F.;Martilli, A.;Fernandez, F. (2019). Heat waves and human well-being in Madrid (Spain). Atmosphere, 10(5), p. 288; https://doi.org/10.3390/atmos10050288

Royé (2017). The effects of hot nights on mortality in Barcelona, Spain. Int J Biometeorology. 61(12): pp. 2127-2140.

Royé, D. (2015). Another Approach for Tropical Nights: Case Studies of Faro, Lisbon and Porto. En: Pina, H. \& Martins F., The overarching issues of the european space: spatial planning and multiple paths to sustainable and inclusive development, Faculdade de Letras da Universidade do Porto, pp. 269-283.

Royé, D. y Fernández García, F. (2017). Biometeorological aspects of hot nights in the urbana rea of Madrid. En Naturaleza, territorio y ciudad en un mundo global. Asociación de Geógrafos Españoles. Madrid, pp. 373-382.

Royé, D. y Martí, A. (2015). Análisis de las noches tropicales en la fachada atlántica de la Península Ibérica. Una propuesta metodológica. Boletin de la Asociacion de Geografos Espanoles, 69, pp. 351-368.

Royé, D.; Martí, A. (2016). Análisis espaciotemporal de las noches cálidas en el litoral mediterráneo de España. En Olcina et al. (coord..), Clima, sociedad, riesgos y ordenación del territorio, Universidad de Alicante y Asociación Española de Climatología, pp. 689-700.

Royé, D.; Sera, F.; Tobías, A.; Lowe, R.; Gasparrini, A.; Pascal, M.; De’Donato, F.; Nunes, B.; Teixeira, J.P. (2021). Effects of hot nights on mortality in South Europe. Epidemiology. In Press.

Sanchez-Guevara, C.; Núñez, M.; Taylor, J.; Mavrogianni, A; Neila, J. (2019). Assessing population vulnerability towards summer energy poverty: Case studies of Madrid and London. Energy and Buildings, Volume 190, Pages 132-143. https://doi.org/10.1016/j.enbuild.2019.02.024

Schleussner, C.; Pfleiderer, P.; Fischer, E. (2017). In the observational record half a degree matters. Nat. Clim. Chang. 7, pp. 460-462. 
Sobrino, J.A.; Oltra-Carrió, R.; Guillem Sòria; Jiménez-Muñoz, J.; Franch, B.; Hidalgo,V.; Mattar, J.; Gómez, A.; De Miguel, E. (2013). Evaluation of the surface urban heat island effect in the city of Madrid by thermal remote sensing, International Journal of Remote Sensing, 34: pp. 9-10, 3177-3192. DOI: 10.1080/01431161.2012.716548

Sousa, PM; Barriopedro, D; Ramos, AM; García-Herrera, R; Espírito-Santo, F; Trigo, RM (2019). Saharan air intrusions as a relevant mechanism for Iberian heatwaves: The record breaking events of August 2018 and June 2019. Weather and Climate Extremes, 26: pp. 100-224. Doi.org/10.1016/j.wace.2019.100224

Vicedo-Cabrera, A.M., Guo, Y., Sera, F. et al. (2018). Temperature-related mortality impacts under and beyond Paris Agreement climate change scenarios. Climatic Change 150, pp. 391-402. https://doi.org/10.1007/s10584-018-2274-3

Xiao, H.; Kopecká, M.; Guo, S.; Guan, Y.; Cai, D.; Zhang, C.; Zhang, X.; Yao, W. (2018). Responses of Urban Land Surface Temperature on Land Cover: A Comparative Study of Vienna and Madrid. Sustainability 10, p. 260. https://doi.org/10.3390/su10020260 
